OPEN ACCESS

Edited by:

Jean-Claude Baron

University of Cambridge,

United Kingdom

Reviewed by:

Catherine Lamy,

Centre Hospitalier

Sainte-Anne, France

Louis R. Caplan,

Harvard Medical School,

United States

*Correspondence:

Qing Peng

qing054@sina.com

Specialty section:

This article was submitted to

Stroke,

a section of the journal

Frontiers in Neurology

Received: 17 July 2021

Accepted: 13 August 2021

Published: 17 September 2021

Citation:

Li Y, Miao Y, Yu M, Zhu Y, Liang Z,

Wang $Z$ and Peng Q (2021) Case Report: PRES-Like Syndrome With

Reversible Cortical Blindness

Associated With Essential

Thrombocythemia.

Front. Neurol. 12:743165

doi: 10.3389/fneur.2021.743165

\section{Case Report: PRES-Like Syndrome With Reversible Cortical Blindness Associated With Essential Thrombocythemia}

\author{
Ying $L_{i}{ }^{1}$, Yuanfeng Miao ${ }^{1}$, Meng Yu ${ }^{1}$, Ying Zhu ${ }^{2}$, Zeyin Liang ${ }^{3}$, Zhaoxia Wang ${ }^{1}$ and \\ Qing Peng ${ }^{1 *}$
}

${ }^{1}$ Department of Neurology, Peking University First Hospital, Beijing, China, ${ }^{2}$ Department of Radiology, Peking University First Hospital, Beijing, China, ${ }^{3}$ Department of Hematology, Peking University First Hospital, Beijing, China

Background: There are few reported cases of posterior reversible encephalopathy syndrome (PRES) combined with essential thrombocythemia (ET). We report a case of PRES-like syndrome in ET.

Case Report: A 60-year-old man with a history of hypertension and thrombocythemia presented with progressive visual loss after waking up; and neurological examination showed pupils were $3 \mathrm{~mm}$ and equally reactive to light, which suggested cortical blindness. Brain magnetic resonance imaging (MRI) revealed restricted diffusion in diffusion-weighted imaging (DWI) in the bilateral parietal and occipital lobes. Routine blood tests revealed a platelet count of $1,044 \times 10^{9} / \mathrm{L}$. ET was diagnosed after exclusion of other causes. Electroencephalography (EEG) showed periodic triphasic waves in the occipital region. The lesions of the parietal and occipital lobes on MRI and periodic triphasic waves of EEG disappeared quickly, and patient's vision returned to normal after the treatment with hydroxyurea and sodium bicarbonate. The patient experienced hallucinatory palinopsia during the recovery of vision.

Conclusion: ET may be a risk factor for PRES.

Keywords: posterior reversible encephalopathy syndrome, reversible cortical blindness, essential thrombocythemia, periodic triphasic waves, palinopsia

\section{INTRODUCTION}

Posterior reversible encephalopathy syndrome (PRES) is characterized by acute neurological symptoms (seizures, encephalopathy, headache, and visual disturbances) in the setting of renal failure, blood pressure fluctuations, cytotoxic drugs, autoimmune disorders, and preeclampsia or eclampsia $(1,2)$. Characteristic radiographic findings include vasogenic edema in the bilateral subcortical regions that resolve within days or weeks. PRES is generally reversible, both radiographically and clinically, and has a favorable prognosis $(1,2)$.

Essential thrombocythemia (ET) is a chronic myeloid neoplasm characterized by symptoms from small or large vessel occlusion and hemorrhage $(3,4)$. In this report, we describe a PRES-like patient who developed bilateral reversible cortical blindness combined with ET. 


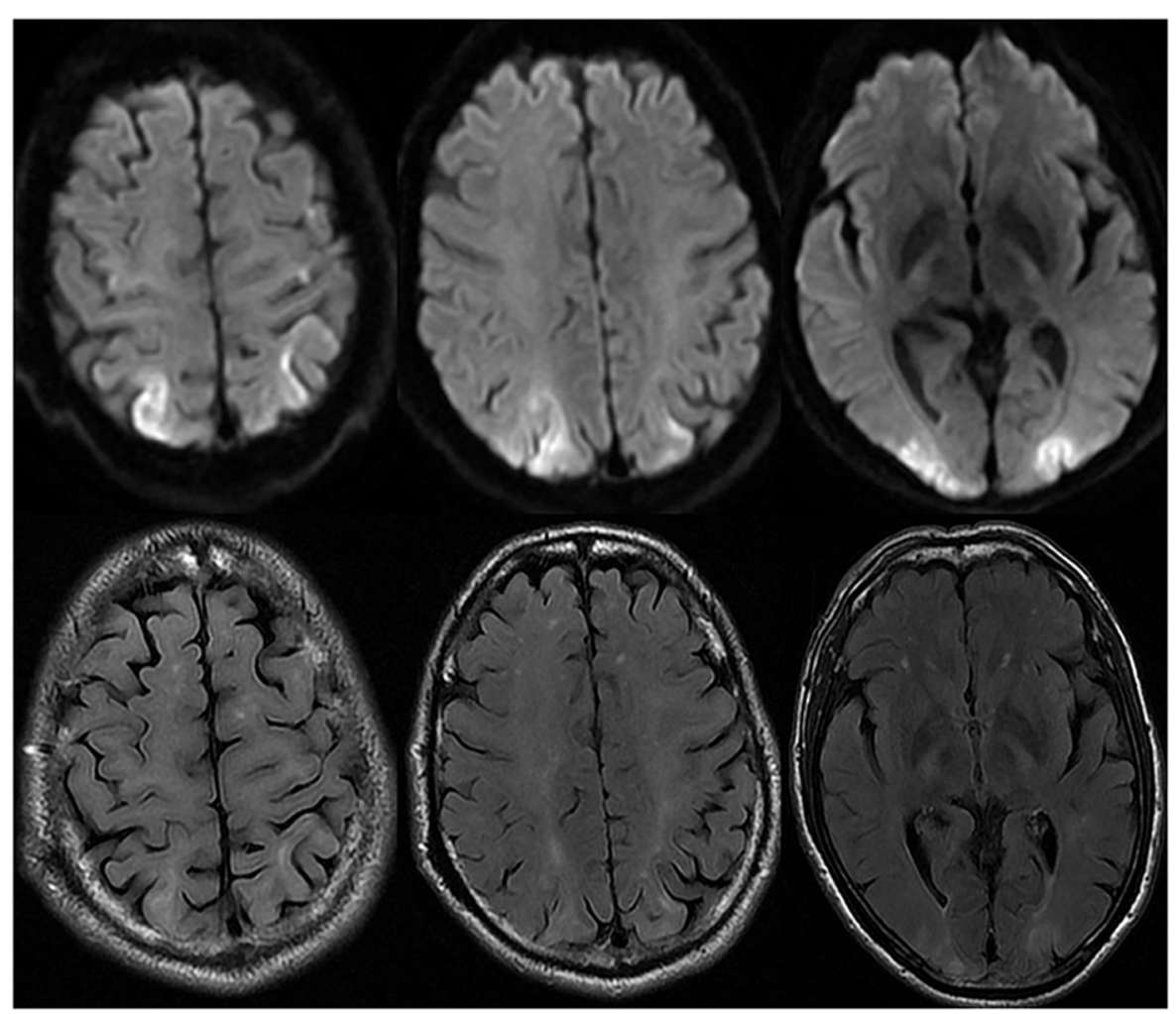

FIGURE 1 | Brain magnetic resonance imaging on the second day after onset. Brain MRI showed diffusion restriction in diffusion-weighted imaging (DWI) and hyperintensity of fluid-attenuated inversion recovery (FLAIR) in the bilateral parieto-occipital lobe on the second day after onset.

\section{CASE REPORT}

A 60-year-old man with a history of hypertension presented to the emergency with sudden visual loss for $7 \mathrm{~h}$ after waking up. Thrombocythemia was found in routine physical examination 7 years ago, and the platelet count was between 400 and $600 \times 10^{9} / \mathrm{L}$. The annual physical examination showed that the liver function, renal function, and abdominal ultrasound were normal. He took aspirin $100 \mathrm{mg} /$ day regularly, but aspirin was stopped 1 week before onset. Platelet count was found to be elevated to $1,044 \times 10^{9} / \mathrm{L}$ after admission. The patient took telmisartan, and the blood pressure was about $120 / 80 \mathrm{mmHg}$ in the past. The patient denied the history of alcoholism. Neurological examination revealed that his visual acuity was severely impaired on both eyes, and he could only recognize waving hands. His pupils were $3 \mathrm{~mm}$ and equally reactive to light. Blood pressure was $128 / 84 \mathrm{mmHg}$. Electrocardiogram showed atrial fibrillation. Urinalysis, liver function, renal function, and ophthalmic examination were normal. Considering that acute cerebrovascular disease could not be excluded, the patient underwent head non-contrast computed tomography and computed tomography angiography (CTA) and computed tomography perfusion (CTP) imaging, which showed no vascular stenosis or cerebral hypoperfusion. After $3 \mathrm{~h}$, the visual acuity of the patient deteriorated, and there was only light perception. There was no obvious change in the second CT scan, and residual contrast agent was found in the venous sinus. However, brain magnetic resonance venography (MRV) showed no venous sinus thrombosis. Brain magnetic resonance imaging (MRI) on the second day after onset (Figure 1) revealed restricted diffusion in diffusion-weighted imaging (DWI) and reduced apparent diffusion coefficient (ADC) in the bilateral parieto-occipital lobes. A bone marrow biopsy showed myeloid granulocytic and megakaryocytic hyperplasia with myelofibrosis. Genetic analysis showed DNMT3A W860R mutation. No mutation was found in $J A K 2, C A L R$, and $M P L$ genes. Contrastenhanced MRI and CT of abdomen revealed stenosis of the trunk and cavernous transformation of the portal vein (CTPV), splenomegaly, splenorenal shunt, and gastric varices. Creactive protein, erythrocyte sedimentation rate, and hemoglobin were normal. Essential thrombocytosis was diagnosed by the hematologist after excluding other causes. The patient developed left upper limb shaking similar to myoclonic seizure within 2 days after the onset of the disease, and then it was relieved spontaneously. Electroencephalography (EEG) showed periodic triphasic waves in the occipital region.

The patient was treated with hydroxyurea and sodium bicarbonate and supportive treatments. On the second day, the patient's vision recovered slightly and had palinoptic afterimages manifesting incorporation of one part of a previously viewed 


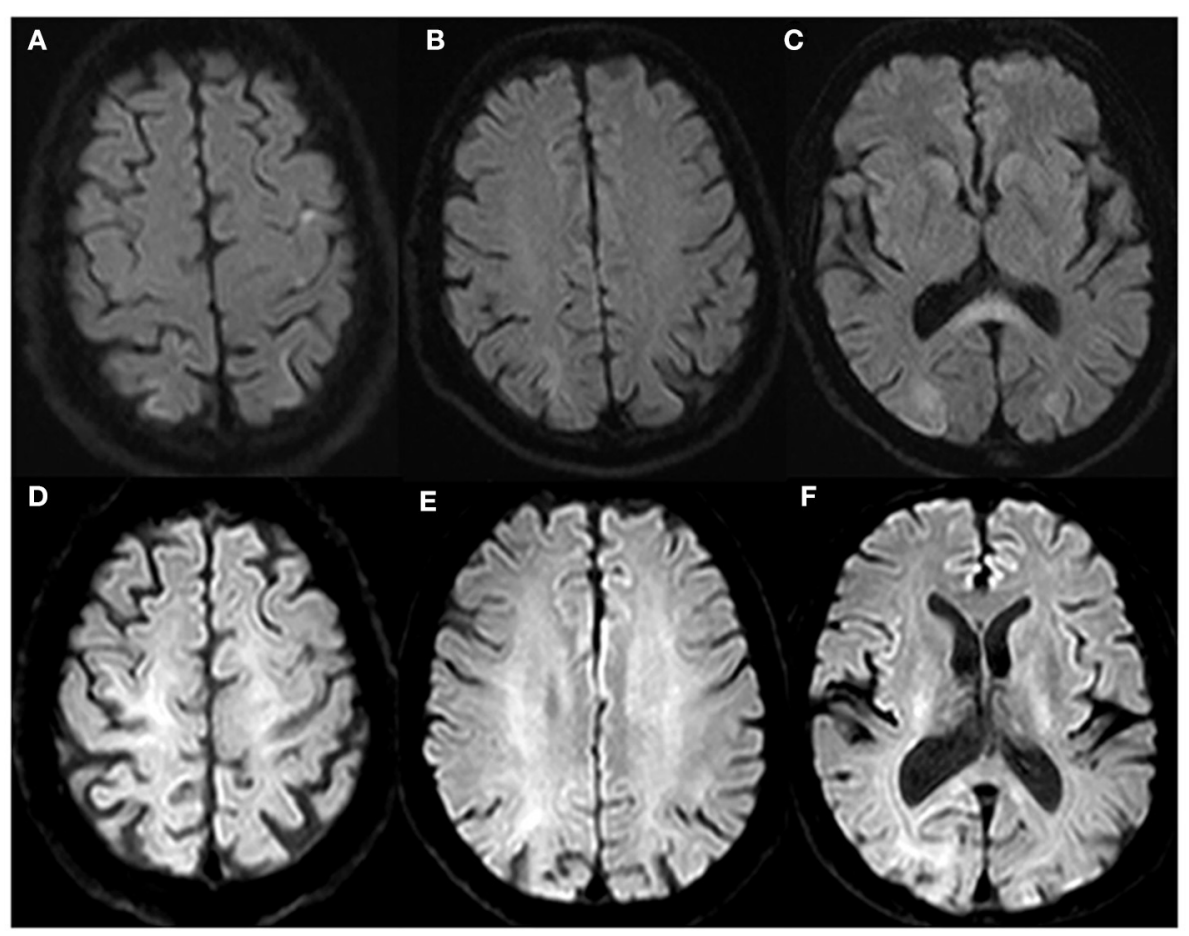

FIGURE 2 | Brain magnetic resonance imaging in follow-up. Brain MRI showed new diffusion-weighted imaging (DWI) diffusion restriction in the splenium of the corpus callosum, and diffusion restriction of the parietal and occipital lobes was lower than before on the eighth day of onset (A-C). Brain MRI revealed the lesions in the bilateral parieto-occipital lobe and splenium of the corpus callosum disappeared, leaving slight atrophy of the bilateral occipital lobe after 4 months (D-F).

object or scene on to the visual image of a contemporaneously viewed second object or scene. The patient's vision returned to normal on the fifth day of onset. The platelet count decreased to 620 to $760 \times 10^{9} / \mathrm{L}$. On the eighth day of onset, the second brain MRI (Figures 2A-C) showed new restricted diffusion in the splenium of the corpus callosum, and hyperintense signal of the parietal and occipital lobes in DWI was lower than before. A diagnosis of PRES was considered. The EEG reexamined 2 weeks after the onset revealed that the triphasic waves disappeared. The patient took aspirin after discharge. Four months later, brain MRI (Figures 2D-F) showed that the abnormal signals in the bilateral parieto-occipital lobes and the splenium of corpus callosum disappeared, leaving slight atrophy of the bilateral occipital lobe with microbleeds.

\section{DISCUSSION}

To our knowledge, this report is the second to document a case of PRES and ET (5). Brain MRI in our patient showed restricted diffusion in DWI and reduced ADC indicating cytotoxic edema, which is consistent with ischemic infarction and different from vasogenic edema of typical PRES. Furthermore, the patient has a stroke-like onset; and several cerebrovascular disease risk factors such as ET, atrial fibrillation and hypertension, and ischemic infarction should be considered. However, no enhancement on enhanced brain MRI, the appearance of lesions in the splenium of the corpus callosum, and the regression of abnormal signals in the occipito-parietal lobes after treatment further support the diagnosis of PRES. According to the literatures, restricted diffusion can be seen on brain MRI in 15-30\% of PRES cases, but large homogeneous regions of restricted diffusion like our patient were rare $(1,2)$. In addition, it is reported that intracranial hemorrhage is common in PRES, complicating 10$25 \%$ of cases, especially ongoing therapeutic anticoagulation and intrinsic coagulopathy (1). Bilateral occipital lobe microbleeds in the follow-up MRI may be related to ET.

There are few reports of PRES combined with ET, so the relationship between PRES and ET is not well-understood. Previous studies suggested that endothelial dysfunction may be involved in the mechanism of PRES in ET patients $(3,5)$. In this patient, the residual contrast agent was still found in the head CT after $3 \mathrm{~h}$ of CTA and MRV excluded venous sinus thrombosis, which suggested that there might be venous stasis caused by ET. Venous stasis may lead to PRES by excessive vasodilation and hyperperfusion. In addition, the patient has a stroke-like onset and whether intravenous thrombolysis could be performed if he arrived at the hospital within 4.5 hours. Previous researches showed that there was an increased risk of major bleeding in patients with platelet count $>1,000 \times 10^{9} / \mathrm{L}(4)$. Henrik et al. (6) found that intravenous thrombolysis in thrombocytosis was associated with increased mortality. Therefore, intravenous thrombolysis should be done cautiously for stroke patients with ET history. 
The patient had a DNMT3A mutation. Soichi et al. (7) found that DNMT3A gene may be associated with myocardial hypertrophy, cardiac dysfunction, and cardiac and renal fibrosis, increasing the risk of cardiovascular disease.

There is no report of triphasic waves in PRES patients so far. The blood ammonia of the patients was $100-125 \mu \mathrm{mol} / \mathrm{L}$ after admission, but the patient had no symptoms of hepatic encephalopathy. The EEG of hepatic encephalopathy mostly showed diffuse triphasic wave changes, which is inconsistent with the focal occipital triphasic waves of the patient (8). In the early stage of onset, the patient developed myoclonic seizure of the left hand, and EEG showed periodic triphasic waves in the bilateral occipital regions, which is similar to the seizure and EEG changes seen in Creutzfeldt-Jakob disease (CJD). Studies have revealed that the EEG patterns are correlated to cerebral imaging findings, and similar EEG changes can occur with different cerebral abnormalities according to the rate of encephalopathy progression, the duration, and so on (9). Therefore, the periodic triphasic waves similar to CJD in this patient may be related to the restricted diffusion of DWI in the cerebral cortex similar to CJD. However, EEG changes in the patient are reversible because of the reversibility of PRES lesions.

Interestingly, the patient experienced hallucinatory palinopsia during the recovery of vision. Visual dysfunction is common in PRES, but there is only one case of palinopsia in PRES patients (10). Palinopsia typically localizes to the non-dominant occipitotemporal cortex, though functional MRI data have suggested its origin in parietal cortical projections to the occipital cortex (11). Its mechanism remains uncertain; cerebral

\section{REFERENCES}

1. Fugate JE, Rabinstein AA. Posterior reversible encephalopathy syndrome: clinical and radiological manifestations, pathophysiology, and outstanding questions. Lancet Neurol. (2015) 14:91425. doi: 10.1016/S1474-4422(15)00111-8

2. Fischer M, Schmutzhard E. Posterior reversible encephalopathy syndrome. $J$ Neurol. (2017) 264:1608-16. doi: 10.1007/s00415-016-8377-8

3. Arellano-Rodrigo E, Alvarez-Larrán A, Reverter JC, Villamor N, Colomer D, Cervantes F. Increased platelet and leukocyte activation as contributing mechanisms for thrombosis in essential thrombocythemia and correlation with the JAK2 mutational status. Haematologica. (2006) 91:169-75.

4. Alvarez-Larran A, Cervantes F, Pereira A, Arellano-Rodrigo E, Pérez-Andreu V, Hernández-Boluda JC, et al. Observation versus antiplatelet therapy as primary prophylaxis for thrombosis in low-risk essential thrombocythemia. Blood. (2010) 116:1205-10. doi: 10.1182/blood-2010-01-263319

5. Zhang Y, Lu Z, Li Y, Wu J, Liu T, Xie X, et al. Posterior reversible encephalopathy syndrome with essential thrombocythemia: a case report. Medicine. (2019) 98:e16759. doi: 10.1097/MD.0000000000016759

6. Gensicke H, Al Sultan AS, Strbian D, Hametner C, Zinkstok SM, Moulin S, etal. Intravenous thrombolysis and platelet count. Neurology. (2018) 90:e6907. doi: 10.1212/WNL.0000000000004982

7. Sano S, Oshima K, Wang Y, Katanasaka Y, Sano M, Walsh K. CRISPRmediated gene editing to assess the roles of Tet2 and Dnmt3a in clonal hematopoiesis and cardiovascular disease. Circ Res. (2018). 123:33541. doi: 10.1161/CIRCRESAHA.118.313225

8. Emmady PD, Murr N. EEG Triphasic Waves. StatPearls. Treasure Island, FL: StatPearls Publishing (2021). hyperperfusion adjacent to the cortex may be implicated in the pathogenesis (11). The distinctive parieto-occipital pattern and hyperperfusion are the causes of hallucinatory palinopsia in PRES patients.

\section{DATA AVAILABILITY STATEMENT}

The original contributions presented in the study are included in the article/supplementary material, further inquiries can be directed to the corresponding author/s.

\section{ETHICS STATEMENT}

The studies involving human participants were reviewed and approved by Peking University First Hospital Ethics Committee. The patients/participants provided their written informed consent to participate in this study. Written informed consent was obtained from the individual's legal guardian/next of kin for the publication of any potentially identifiable images or data included in this article.

\section{AUTHOR CONTRIBUTIONS}

YL: study concept, data collection, and drafting of the manuscript. YM, MY, YZ, ZL, and ZW: data evaluation and manuscript revision. QP: study concept, data collection, and critical revision. All authors contributed to the article and approved the submitted version.

9. Kaplan PW, Rossetti AO. EEG patterns imaging correlations in encephalopathy: encephalopathy part II. J Clin Neurophysiol. (2011) 28:233-51. doi: 10.1097/WNP.0b013e31821c33a0

10. Ghosh R, Lahiri D, Dubey S, Ray BK, Benito-León J. Hallucinatory Palinopsia in COVID-19-Induced Posterior Reversible Encephalopathy Syndrome. J Neuroophthalmol. (2020) 40:523-6. doi: 10.1097/WNO.000000000000 1135

11. Norton JW, Corbett JJ. Visual perceptual abnormalities: hallucinations and illusions. Semin Neurol. (2000) 20:111-21. doi: 10.1055/s-2000-6837

Conflict of Interest: The authors declare that the research was conducted in the absence of any commercial or financial relationships that could be construed as a potential conflict of interest.

Publisher's Note: All claims expressed in this article are solely those of the authors and do not necessarily represent those of their affiliated organizations, or those of the publisher, the editors and the reviewers. Any product that may be evaluated in this article, or claim that may be made by its manufacturer, is not guaranteed or endorsed by the publisher.

Copyright () $2021 \mathrm{Li}$, Miao, Yu, Zhu, Liang, Wang and Peng. This is an open-access article distributed under the terms of the Creative Commons Attribution License (CC $B Y)$. The use, distribution or reproduction in other forums is permitted, provided the original author(s) and the copyright owner(s) are credited and that the original publication in this journal is cited, in accordance with accepted academic practice. No use, distribution or reproduction is permitted which does not comply with these terms. 